\title{
Mechanical properties of all-suture anchors for rotator cuff repair
}

\section{N. S. Nagra, \\ N. Zargar, \\ R. D. J. Smith, \\ A. J. Carr}

NDORMS, University

of Oxford, Botnar

Research Centre, Nuffield Orthopaedic Centre, Windmill

Road, Headington,

Oxford, United

Kingdom
N. S. Nagra, MB ChB, MSc Med Sci (TERM), DPhil Candidate, NDORMS, University of Oxford, Botnar Research Centre, Nuffield Orthopaedic Centre, Windmill Road, Headington, Oxford, UK and, Oxford University Clinical Academic Graduate School, Medical Sciences Divisional Office, Level 3, John Radcliffe Hospital, Oxford, UK.

N. Zargar, DPhil, PostDoctoral Researcher, NDORMS,

R. D. J. Smith, AB, DPhil, Medical Student, NDORMS, and

A. J. Carr, ChM, DSc, FRCS, FMedSci, Head of Department, Professor of Orthopaedics, NDORMS, University of Oxford, Botnar Research Centre, Nuffield Orthopaedic Centre, Windmill Road, Headington, Oxford, UK.

Correspondence should be sent to N.S. Nagra; email: navraj.nagra@ ndorms.ox.ac.uk

doi: $10.1302 / 2046-3758.62 . B \mid R-$ 2016-0225.R1

Received: 23 August 2016; Accepted: 8 December 2016 Bone Joint Res 2017;6:82-89.

\section{Objectives}

All-suture anchors are increasingly used in rotator cuff repair procedures. Potential benefits include decreased bone damage. However, there is limited published evidence for the relative strength of fixation for all-suture anchors compared with traditional anchors.

\section{Materials and Methods}

A total of four commercially available all-suture anchors, the ' $Y$-Knot' (ConMed), Q-FIX (Smith \& Nephew), ICONIX (Stryker) and JuggerKnot (Zimmer Biomet) and a traditional anchor control TWINFIX Ultra PK Suture Anchor (Smith \& Nephew) were tested in cadaveric human humeral head rotator cuff repair models $(n=24)$. This construct underwent cyclic loading applied by a mechanical testing rig (Zwick/Roell). Ultimate load to failure, gap formation at 50, 100, 150 and 200 cycles, and failure mechanism were recorded. Significance was set at $\mathrm{p}<0.05$.

\section{Results}

Overall, mean maximum tensile strength values were significantly higher for the traditional anchor (181.0 N, standard error (SE) 17.6) compared with the all-suture anchors (mean 133.1 N SE 16.7) $(p=0.04)$. The JuggerKnot anchor had greatest displacement at 50, 100 and 150 cycles, and at failure, reaching statistical significance over the control at 100 and 150 cycles (22.6 mm SE 2.5 versus $12.5 \mathrm{~mm}$ SE 0.3 ; and $29.6 \mathrm{~mm}$ SE 4.8 versus $17.0 \mathrm{~mm}$ SE $0.7)$. Every all-suture anchor tested showed substantial $(>5 \mathrm{~mm}$ ) displacement between 50 and 100 cycles (6.2 to 14.3 ).

All-suture anchors predominantly failed due to anchor pull-out ( $95 \%$ versus $25 \%$ of traditional anchors), whereas a higher proportion of traditional anchors failed secondary to suture breakage.

\section{Conclusion}

We demonstrate decreased failure load, increased total displacement, and variable failure mechanisms in all-suture anchors, compared with traditional anchors designed for rotator cuff repair. These findings will aid the surgeon's choice of implant, in the context of the clinical scenario.

\section{Cite this article: Bone Joint Res 2017;6:82-89.}

Keywords: All-suture anchors, Rotator cuff, Mechanical properties, Failure load, Total displacement, Failure mechanisms, Shoulder

\section{Article focus}

- To compare 'all-suture' anchors with traditional anchors within a human, cadaveric, humeral head rotator cuff repair model.

- To determine ultimate load to failure, gap formation, and mechanism of failure of anchors tested using this model.

\section{Key messages}

- Traditional anchors had higher maximum tensile strength values compared with the mean of all 'all-suture' anchors tested.
- Displacement profiles varied between traditional and 'all-suture' anchors, with greater displacement generally reported in some, but not all 'all-suture' anchors.

- Failure mechanisms varied between anchor types with 'all-suture' anchors.

\section{Strengths and limitations}

n This is the first study to compare the four commercially available 'all-suture' anchors with a traditional anchor control using a human cadaveric model. 
- Further work requires larger sample sizes with a model capable of reproducing in vivo conditions and multidirectional forces, with radiographic bone density controls.

\section{Introduction}

Anchorage of sutures in the repair of rotator cuff (RC) tears has traditionally made use of large, screw-shaped anchors consisting of either metal or plastic polymer materials to secure sutures into bone. However, over the last decade, a novel fixation technique has emerged. Initially designed for labral and capsular tear repairs, 'allsuture' anchors are increasingly being used in RC tear repair surgery. Placement of all-suture anchors generally involves drilling a small pilot hole into the bone, and subsequently placing suture material in a latent configuration attached to a catheter device, which allows the suture to expand in the cancellous bone under the cortex as the catheter is removed.

$\mathrm{RC}$ repair is a procedure which has a reported failure rate of between $13 \%^{1}$ and $94 \%$ (as defined by re-rupture post repair). ${ }^{2}$ Some authors have claimed that all-suture anchors facilitate easier revision after surgical failure because they are designed to preserve bone stock and improve post-operative imaging after surgery. ${ }^{3}$

Previous studies have endeavoured to look into the mechanical properties of all-suture anchors and compare these with traditional bone anchors. ${ }^{4-9}$ However, such studies have been incomplete: studying only one allsuture anchor at a time; ${ }^{7}$ studying only mechanical properties such as maximum tensile strength; ${ }^{4}$ or using less relevant canine, ${ }^{9}$ bovine, ${ }^{6}$ porcine, ${ }^{4,5}$ and human tibial ${ }^{8}$ models to demonstrate the mechanical properties of allsuture anchors in the human humeral head. Furthermore, studies have shown the maximum tensile strength of anchors to range from $49 \mathrm{~N}$ to $66 \mathrm{~N}$ in one paper, 8 to 760 $\mathrm{N}$ in another, ${ }^{5}$ highlighting the need for a comprehensive evaluation of all commercially available all-suture anchors in comparison with traditional anchors.

The aim of this study was to compare the mechanical properties (failure load and cyclic displacement) and failure mechanisms of all-suture anchors with those of a traditional suture anchor in cadaveric human humeral heads. The null hypothesis of the study was that the mechanical properties and failure mode of novel commercial all-suture anchors would be the same as those of a traditional bone anchor.

\section{Materials and Methods}

Cadaveric sample acquisition. A total of 24 cadaveric samples were obtained from the Department of Physiology, Anatomy and Genetics, University of Oxford (Oxford, United Kingdom). The humeral heads were dissected from cadaveric donors and overlying soft-tissue was debrided to expose the bone surface over the greater tuberosity. The humerus was isolated from the distal upper limb $10 \mathrm{~cm}$ below its surgical neck. Macroscopic examination was used to exclude obvious deformity and bony defects within samples by two authors (NSN, RDJS). All samples were kept hydrated in $0.9 \%$ saline prior to transfer for anchor insertion and subsequent mechanical testing ( $n=24,12$ matched pairs, age range 58 to $96,4: 2$ ratio of men:women).

Anchors tested. A review of the literature and a search through the procurement department of our tertiary referral centre hospital was conducted to determine current commercially available all-suture anchors (accurate as of April 2016), of which four were found. All four types of commercially available all-suture anchors and one traditional plastic anchor were obtained from the manufacturers. All-suture anchors tested included the Y-Knot (ConMed, New York, New York), Q-FIX (Smith \& Nephew, London, United Kingdom), ICONIX (Stryker, Kalamazoo, Michigan) and JuggerKnot (Zimmer Biomet, Warsaw, Indiana). A commonly used traditional plastic anchor (TWINFIX Ultra PK Suture Anchors, Smith \& Nephew) was used as a comparator and control. All tested anchors had polyethylene (flat braided ultra-high molecular weight polyethylene (UHMWPE); Y-Knot) or braided polyester \#2 sutures for tendon interfaces.

All described anchors are indicated for both open and arthroscopic RC repair. Variations in size and suture type are available for other procedures including repair of Bankart and SLAP (superior labral tears from anterior to posterior) lesions, biceps tenodesis, acromioclavicular joint dislocation reconstruction, deltoid repair and capsulolabral repair. However, these sizes were not tested.

ICONIX 3 anchors are composed of three strands of 'Force Fiber' suture (UHMWPE, polypropylene and nylon) enclosed in a sheath structure, which bunches under tension to form a suture-anchor (UHMWPE). The ICONIX is designed to deploy under tension applied by the surgeon, with a minimum of $10 \mathrm{~N}$ of force. Initially, a guide is placed over the greater tuberosity before a self-tapping $2.3 \mathrm{~mm}$ awl is engaged through the guide and advanced to break through the cortex and displace underlying bone until a positive stop is achieved. The awl is removed and the anchor is placed through the guide and, using a mallet, advanced until a positive stop is achieved. The suture ends are untethered from the anchor inserter, and both the inserter and guide are removed. It should be noted that other sizes of ICONIX anchor are available and indicated for RC repair surgery, however, literature published by the manufacturer states that the ICONIX 3 is the strongest (defined by maximum tensile strength) anchor within the range. ${ }^{10}$

Q-FIX anchors consist of a braided polyester anchor implant encompassing two \#2 'MagnumWire' sutures (UHMWPE). The implant is placed by pushing a drill guide and associated obturator through soft tissues until 


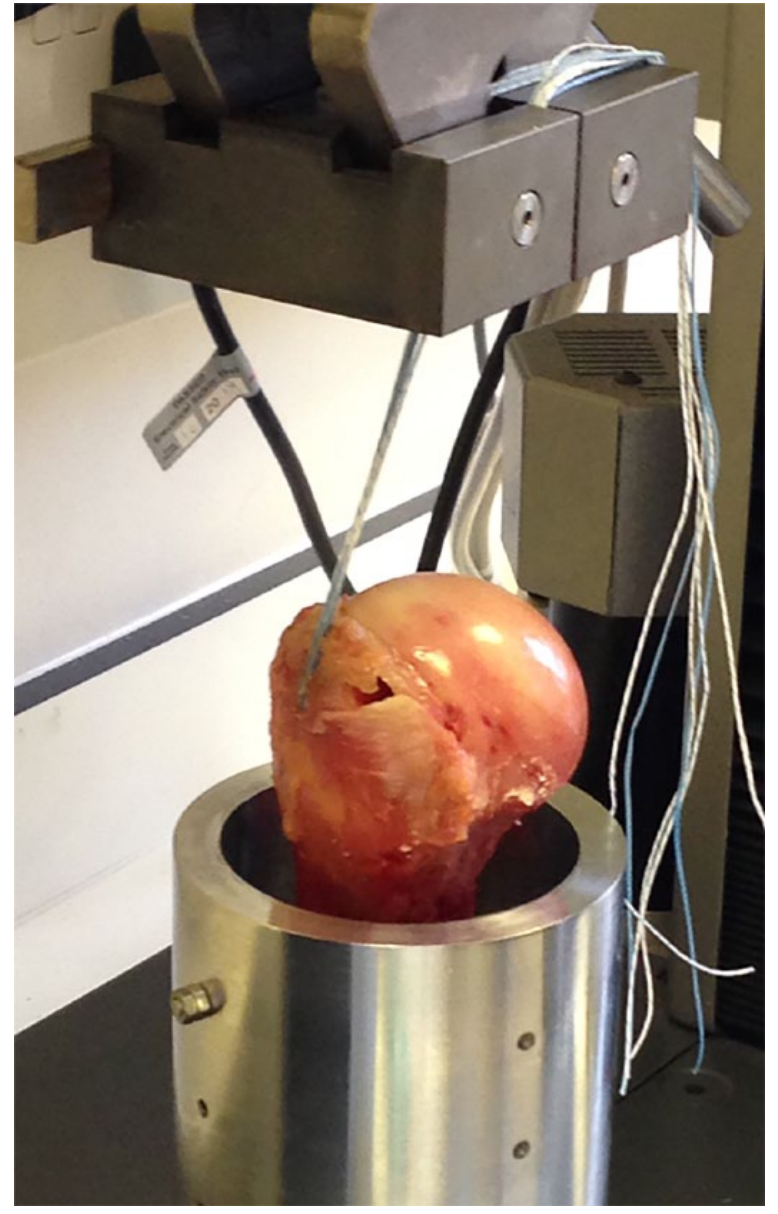

Fig. 1

The experimental construct used to determine maximum tensile strength and displacement.

they approximate cortex. Once the soft tissue has been navigated, the obturator is removed, leaving the drill guide overlying the cortex. A $3 \mathrm{~mm}$ drill bit is placed through the drill guide to breach the cortex and create a bone hole - this is terminated when the drill bottoms onto the guide and no further progress can be made. The drill is subsequently removed and replaced with the Q-FIX implant inserter which goes into the guide. Once the implant inserter is fully engaged (meaning the distal end of the inserter is within the newly formed bone tunnel), the 'activation knob' of the implant inserter is then rotated clockwise about its axis to deploy the spherical suture-anchor to a reported internal force of $140 \mathrm{~N} .{ }^{11}$ Suture ends are separated from the inserter and the inserter and guide are removed.

Y-Knot anchors are made of three 'HiFi' sutures (UHMWPE, polypropylene and nylon), contained within a 'HiFi' flat braided suture tape (UHMWPE). There are two possible insertion techniques for placement of the Y-Knot. The first, a self-tapping technique, involves placing the tip of the inserter on to the cortex and subsequently using a mallet to impact the inserter with suture attached into the bone. Circumferential laser-marks indicate the range of the depth to which the inserter can be pressed. A second option, for harder bone, is to create a $2.8 \mathrm{~mm}$ pilot hole before insertion of the suture in a manner similar to that already described. Thereafter, sutures are unwound from the inserter and the construct is removed. All suture strands are then pulled and the anchor is compressed against the cortical bone.

JuggerKnot anchors are formed from two \#2 'MaxBraid' (UHMWPE) sutures engaged on an insertion device within a braided polyester anchor implant. After initially boring through the cortex with a $2.9 \mathrm{~mm}$ drill, the anchor is inserted and deployed under tension to cause the braided polyester to gather under the cortex and secure the anchor.

Loading protocol. All suture anchors were placed into the anterior and proximal aspect of the greater tuberosity, 1 $\mathrm{cm}$ posterior to the bicipital groove, in accordance with the manufacturer's instructions using suggested insertion tools and techniques after instruction from a manufacturer's representative. The humeral shaft was pierced along its length and secured within a custom-designed platform with screws. The suture threads emerging from the humeral head were secured within the upper clamps of the rig, with no recorded failure in any repeats at the suture-vice interface (Fig. 1).

Cyclical loading using a previously published protocol to simulate the rehabilitation phase of post-rotator cuff tendon repair ${ }^{12}$ was used (see supplementary material). Tensile testing to failure was then performed using a Zwick/Roell tensile testing machine (Zwick Roell Group, Ulm, Germany) and a $10 \mathrm{kN}$ load cell, with a clamp-toclamp distance of $100 \mathrm{~mm}$. An initial $10 \mathrm{~N}$ tension was placed on the anchors to ensure proper deployment of the subcortical segment of the all-suture anchors and to prevent loading artefacts. ${ }^{6}$ Maximum tensile strength and displacement of the construct were measured by means of the experimental configuration demonstrated (Fig. 1), using the intrinsic force and distance calculators of the tensile testing machine (testXpert II; Zwick Roell Group) which calculates tensile force deployed by the load cell, and change in distance between the two clamps. Mechanism of failure (suture or interface) was also recorded. A minimum of four repeats were conducted for each implant $(n \geq 4)$.

Cadaveric samples were distributed such that a combination of left and right humeral heads from the same patient were used with different anchors, and different anchors were used with different cadaver ages to ensure matched ages and distribution amongst samples.

Statistical analysis. Kruskal-Wallis one-way analyses were performed to compare the tensile mechanical properties of the different commercial suture anchors and traditional bone anchor to determine initial variance. Post hoc analysis was performed using the Dunn test. Comparisons of 


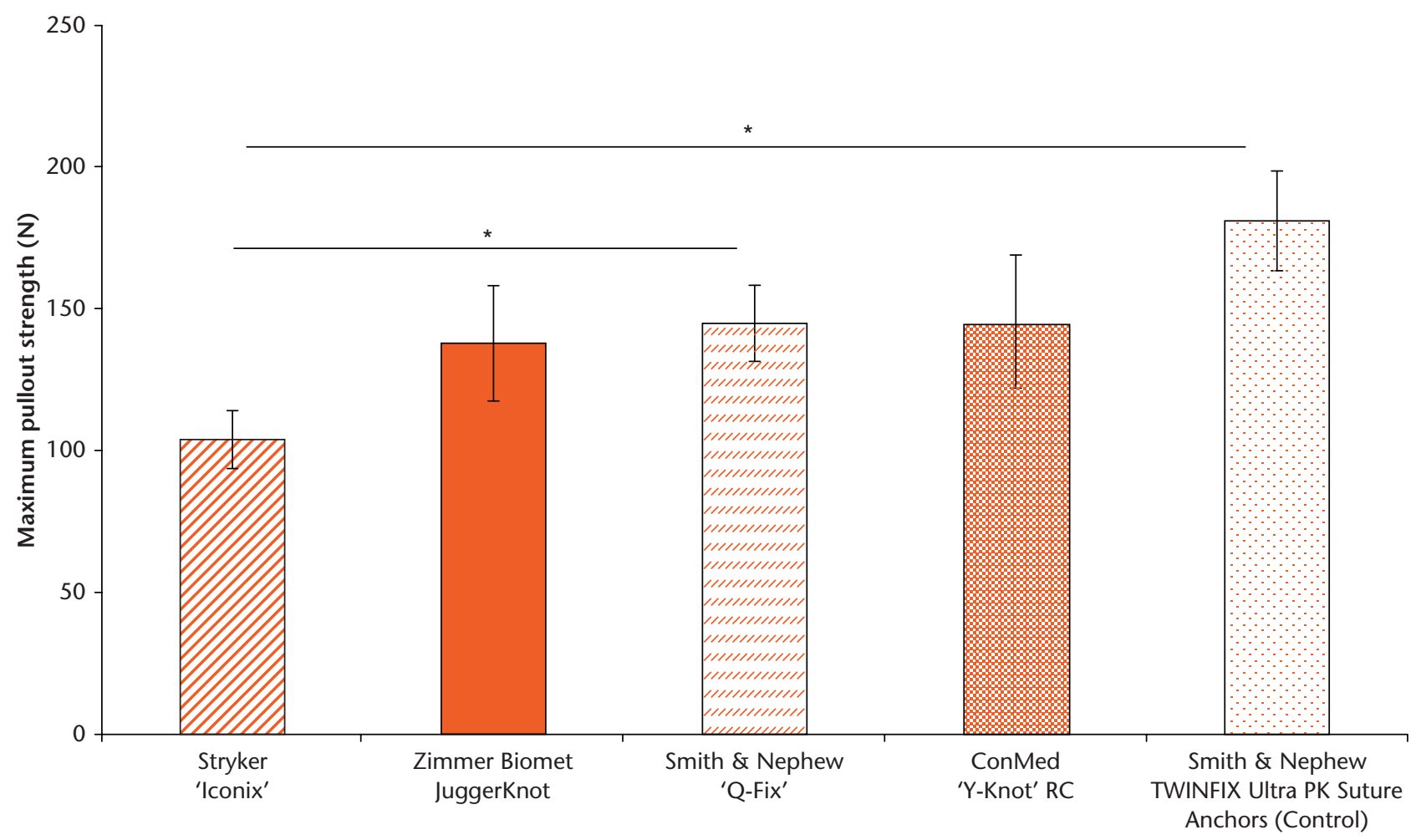

Anchor brand and name

Fig. 2

Maximum tensile strength of all-suture anchors and a traditional bone anchor as assessed to failure. Error bars represent standard error. Significance $\mathrm{p}<0.05\left({ }^{*}\right)$.

all-suture anchors against the traditional bone anchor were made using Student's $t$-test. All statistical tests had a significance level of $p<0.05$ (and are reported to one significant figure below 0.05 ) and were performed using GraphPad Prism 6 software (GraphPad Software Inc., La Jolla, California).

\section{Results}

Tensile strength. Statistically significant variance was noted between anchor types and between all-suture anchors with regard to maximum failure and displacement (Tables I13,14 and II).

Maximum tensile strength was significantly greater in the control anchor (mean and standard error: $181.0 \mathrm{~N}$, SE 17.6) and Q-FIX (144.9 N, SE 13.4) over the ICONIX (103.9 N, SE 10.1) anchors $(p=0.02, p=0.04$ and $p=$ 0.03 , respectively) (Table I, Fig. 2). JuggerKnot and Y-Knot anchors (137.8 SE $20.3 \mathrm{~N}$ and 145.8 SE $23.1 \mathrm{~N}$, respectively) did not have significantly different tensile strength values when compared with other anchors.

The mean maximum tensile strength value was significantly higher for the traditional anchor (181.0 N, SE 17.6) compared with the all-suture anchors (mean $133.1 \mathrm{~N}$, SE 16.7; $p=0.04$ ).

Gap formation of construct. Displacement, after initial application of load, was recorded during cyclic loading. Comparisons were not made at 200 cycles as the majority
Table I. Mechanical properties and failure mechanisms observed of tested anchors ${ }^{13,14}$

\begin{tabular}{|c|c|c|c|}
\hline Studied anchor & $\mathbf{n}$ & $\begin{array}{l}\text { Maximum tensile } \\
\text { force, mean (sE) }\end{array}$ & $\begin{array}{l}\text { Failure } \\
\text { mechanism (n) }\end{array}$ \\
\hline Human SST enthesis & $n / a$ & $784^{13}$ to $1978^{14}$ & $\mathrm{n} / \mathrm{a}$ \\
\hline $\begin{array}{l}\text { ConMed (New York, } \\
\text { New York) Y-Knot RC }\end{array}$ & 5 & 145.8 (SE 23.1) & Anchor pull-out (5) \\
\hline $\begin{array}{l}\text { Smith \& Nephew } \\
\text { (London, United } \\
\text { Kingdom) Q-FIX }\end{array}$ & 5 & 144.9 (SE 13.4) & Anchor pull-out (5) \\
\hline $\begin{array}{l}\text { Stryker (Kalamazoo, } \\
\text { Michigan) ICONIX }\end{array}$ & 5 & $103.9($ SE 10.1) & Anchor pull-out (5) \\
\hline $\begin{array}{l}\text { Zimmer Biomet (Warsaw, } \\
\text { Indiana) JuggerKnot }\end{array}$ & 5 & 137.8 (SE 20.3) & $\begin{array}{l}\text { Anchor pull-out (4) } \\
\text { Suture failure (1) }\end{array}$ \\
\hline $\begin{array}{l}\text { Smith \& Nephew TWINFIX } \\
\text { Ultra PK Suture Anchors }\end{array}$ & 4 & $181.0($ SE 17.6) & $\begin{array}{l}\text { Eyelet fracture (1) } \\
\text { Anchor pull-out (1) } \\
\text { Suture failure (2) }\end{array}$ \\
\hline
\end{tabular}

SE, standard error; SST, supraspinatus tendon; $n / a$, not applicable

of anchors failed before this point (88.2\% of all-suture anchors). The JuggerKnot anchor had the greatest displacement at 50, 100 and 150 cycles and also at failure, though this did not reach statistical significance over any other all-suture anchors (Table II) apart from the Q-FIX anchor at failure (Fig. 3). The JuggerKnot anchor did have significantly greater displacement over control anchors at 100 and 150 cycles $(22.6 \mathrm{~mm}$, SE 2.5 versus $12.5 \mathrm{~mm}$, SE $0.3, p=0.01$; and $29.6 \mathrm{~mm}$, SE 4.8 versus $17.0 \mathrm{~mm}$, SE 0.7, $p=0.03$, respectively). 
Table II. Displacement values ( $\mathrm{mm}$ ) of tested anchors. ICONIX anchors did not reach 150 cycles and Q-FIX anchors did not reach 200 cycles, therefore data are not included; standard error (SE) at 200 cycles is not described for all anchors as anchors failed before this point

\begin{tabular}{|c|c|c|c|c|c|c|c|}
\hline Studied anchor & $\mathbf{n}$ & $\begin{array}{l}\text { Mean } \\
\text { displacement } \\
\text { at } 50 \text { cycles, } \\
\text { mm (sE) }\end{array}$ & $\begin{array}{l}\text { Mean } \\
\text { displacement } \\
\text { at } 100 \text { cycles, } \\
\text { mm (SE) }\end{array}$ & $\begin{array}{l}\text { Mean } \\
\text { displacement } \\
\text { at } 150 \text { cycles, } \\
\text { mm (SE) }\end{array}$ & $\begin{array}{l}\text { Mean } \\
\text { displacement at } \\
200 \text { cycles, } \mathbf{m m}\end{array}$ & $\begin{array}{l}\text { Mean total } \\
\text { displacement, } \\
\text { mm (sE) }\end{array}$ & $\begin{array}{l}\text { Mean number } \\
\text { of cycles at } \\
\text { failure (SE) }\end{array}$ \\
\hline $\begin{array}{l}\text { ConMed (New York, New York) } \\
\text { Y-Knot RC }\end{array}$ & 5 & 9.2 (SE 1.2) & 15.1 (SE 2.1) & 20.9 (SE 3.7) & 27.3 & 23.6 (SE 2.2) & 151 (SE 19) \\
\hline $\begin{array}{l}\text { Smith \& Nephew (London, } \\
\text { United Kingdom) Q-FIX }\end{array}$ & 5 & 6.9 (SE 2.2) & 11.8 (SE 3.4) & 14.6 (SE 4.5) & $\begin{array}{l}\text { Construct failure } \\
\text { prior to data point }\end{array}$ & 20.3 (SE 3.3) & 161 (SE 16) \\
\hline $\begin{array}{l}\text { Stryker (Kalamazoo, Michigan) } \\
\text { ICONIX }\end{array}$ & 5 & 8.4 (SE 0.7) & 17.9 (SE 1.9) & $\begin{array}{l}\text { Construct failure } \\
\text { prior to data point }\end{array}$ & $\begin{array}{l}\text { Construct failure } \\
\text { prior to data point }\end{array}$ & 22.7 (SE 2.2) & 112 (SE 12) \\
\hline $\begin{array}{l}\text { Zimmer Biomet (Warsaw, } \\
\text { Indiana) JuggerKnot }\end{array}$ & 5 & 10.3 (SE 1.5) & 22.6 (SE 2.5) & 29.6 (SE 4.8) & 22.9 & 33.7 (SE 4.0) & 146 (SE 19) \\
\hline $\begin{array}{l}\text { Smith \& Nephew TWINFIX } \\
\text { Ultra PK Suture Anchors }\end{array}$ & 4 & 7.5 (SE 0.5) & 12.5 (SE 0.3) & 17.0 (SE 0.7) & 21.5 & 19.7 (SE 1.0) & 175 (SE 9) \\
\hline
\end{tabular}

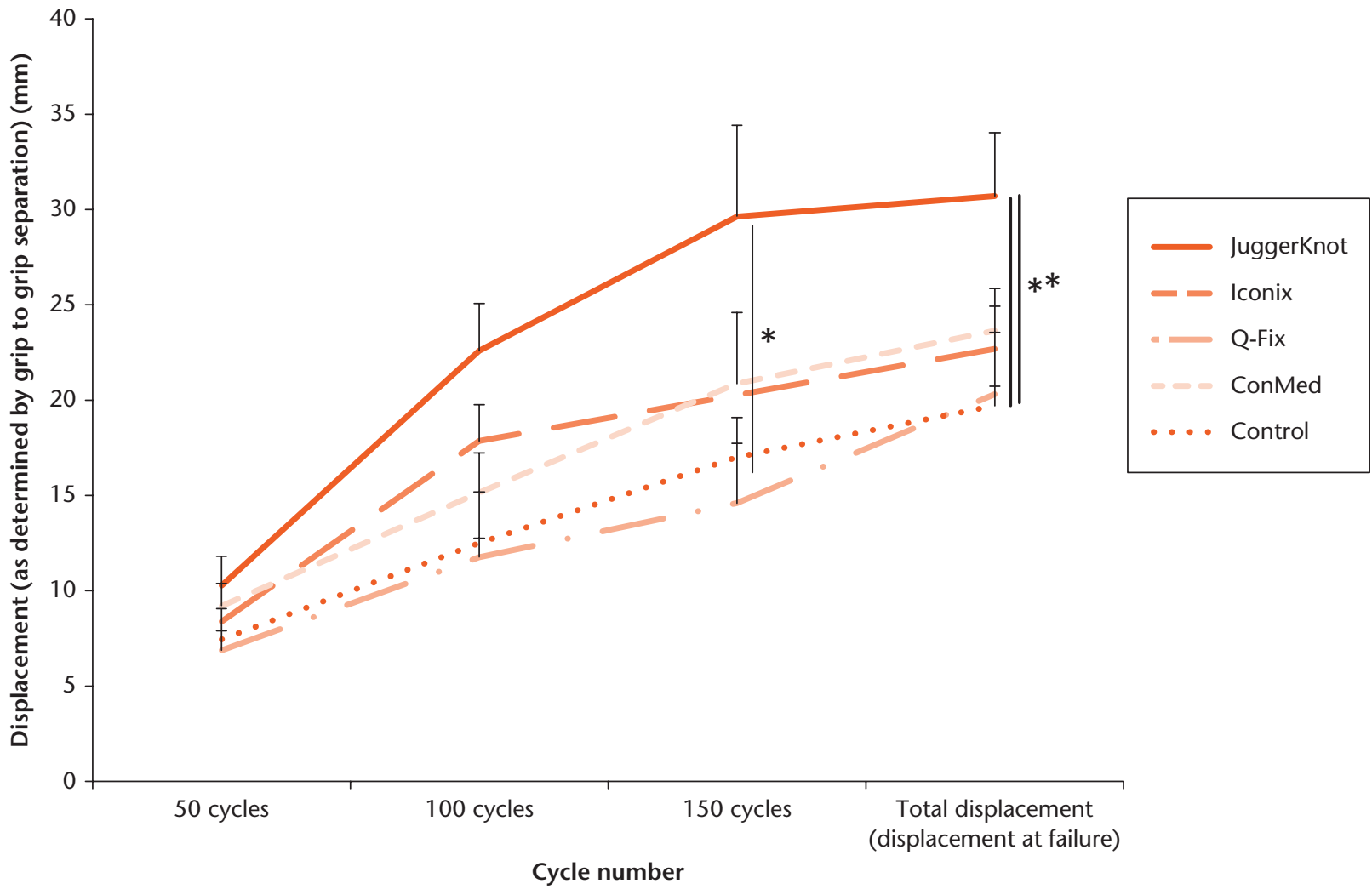

Fig. 3

Displacement of all-suture anchors as determined by changes in grip-to-grip distance over increasing cycle numbers and eventually to failure. Error bars represent upper limits of standard error to demonstrate maximum displacement. Significance $p<0.05\left(^{*}\right)$.

With the exception of the Q-FIX (4.9 mm, SE 1.2), allsuture anchors showed significant displacement between 50 and 100 cycles (defined as $>5 \mathrm{~mm}$ during loading, range 6.2 to 14.3$)^{15,16}$ (Table II, Fig. 3).

Moreover, all anchors demonstrated significant displacement between 0 and 50 cycles (range 6.9 to 9.2) and also between 100 and 150 cycles (range 5.8 to 6.7), except the ICONIX, which did not reach this cycle number.

The JuggerKnot implant had the greatest increase in displacement between 50 and 100 cycles, with mean displacement of $14.3 \mathrm{~mm}$ (SE 2.2), which was significantly greater than that of the Q-FIX implant $(p=0.03$ ). Otherwise, there was no significant variation between displacement values of the anchors.

Additional analysis of displacement was performed to determine changes in displacement (differentials) after the first 50 cycles to accommodate initial anchor settling. Displacement between 50 and 100 cycles, 50 and 150 cycles, and 50 cycles to failure were determined (Table III).

Between 50 and 100 cycles, the JuggerKnot demonstrated the greatest mean displacement (12.3 mm, SE 2.6), 
Table III. Mean displacement differential values and standard error (SE) (mm) of tested anchors between 50 cycles and: 100 cycles, 150 cycles, and failure. ICONIX anchors did not reach 150 cycles, therefore these data are not included

\begin{tabular}{|c|c|c|c|c|}
\hline Studied anchor & $\mathbf{n}$ & $\begin{array}{l}\text { Mean displacement } \\
\text { differential between } 50 \\
\text { and } 100 \text { cycles, } \mathbf{m m} \text { (sE) }\end{array}$ & $\begin{array}{l}\text { Mean displacement } \\
\text { differential between } 50 \\
\text { and } 150 \text { cycles, mm (SE) }\end{array}$ & $\begin{array}{l}\text { Mean displacement } \\
\text { differential between } 50 \\
\text { cycles and failure, } \mathbf{m m} \text { (SE) }\end{array}$ \\
\hline ConMed (New York, New York) Y-Knot RC & 5 & 6.2 (SE 0.7) & 11.7 (SE 2.2) & $14.4($ SE 1.6$)$ \\
\hline Smith \& Nephew (London, United Kingdom) Q-FIX & 5 & 4.9 (SE 1.2) & $9.8(\mathrm{SE} 3.7)$ & 13.4 (SE 2.3) \\
\hline Stryker (Kalamazoo, Michigan) ICONIX & 5 & 9.1 (SE 1.9) & $\begin{array}{l}\text { Construct failure prior } \\
\text { to data point }\end{array}$ & 14.3 (SE 1.8) \\
\hline Zimmer Biomet (Warsaw, Indiana) JuggerKnot & 5 & $12.3(\mathrm{SE} 2.6)$ & $21.0($ SE 3.8) & 20.4 (SE 3.4) \\
\hline Smith \& Nephew TWINFIX UItra PK Suture Anchors & 4 & $5.1(\mathrm{SE} 0.4)$ & $9.6(\mathrm{SE} 1.1)$ & $12.3(\mathrm{SE} 1.0)$ \\
\hline
\end{tabular}

which reached statistical significance against the control and Q-Fix anchors (5.1 mm, SE 0.4 and $4.9 \mathrm{~mm}$, SE 1.2; $\mathrm{p}=$ 0.03 and $p=0.02$, respectively). A similar pattern was found between cycles 50 and 150 , whereby the JuggerKnot anchor had the greatest displacement (21.0 mm, SE 3.8), which was statistically significant against the control anchor (9.6 mm, SE 1.1; p = 0.02). However, when comparing displacement between 50 cycles and failure, there was no statistically significant variation between anchors.

Failure mechanism. Experimental constructs failed by a variety of mechanisms, with the majority of all-suture anchors failing by anchor pull-out, whereby anchor integrity was maintained. However, cortical failure occurred in $95 \%$ of all-suture anchors and $25 \%$ of traditional bone anchors. A higher proportion of traditional bone anchors failed due to the suture itself breaking, suggesting greater strength of anchor fixation ( $5 \%$ of all-suture anchors versus $50 \%$ of traditional bone anchors).

\section{Discussion}

This study compared the mechanical properties of currently marketed commercial all-suture anchors, using a biologically representative ex vivo model, with a traditional bone anchor comparator. The study found that the load-to-failure was lower and displacement was significantly greater in some all-suture anchors compared with a control traditional anchor. These findings are consistent with previously published literature in non-human specimens. ${ }^{4,6,9}$ The mechanical properties of bone vary according to structure (particularly the thickness of the cortex), species of animal and site tested, thus adding weight to the argument that tests to determine strength of implants should be standardised in an anatomically and biologically representative model. ${ }^{5}$ This study is representative of the environment into which these anchor types are being placed, more so than previous work, which has looked at retention strength in animal, polyurethane and human diaphyseal bone. $4,5,6,17$

Although we discuss here load-to-failure and displacement as important mechanical characteristics to define the properties of anchors, these in themselves may be too simplistic to determine the value of implants for RC repair. Due to their smaller size, a greater number of allsuture anchors can be placed in the same area of bone, allowing for greater strength of fixation and decreased gap formation. ${ }^{6,12}$ Indeed, one study showed that no demonstrable difference was detected between the pull-out strengths of two all-suture anchors (JuggerKnot $1.4 \mathrm{~mm}$ ) and one traditional anchor (PEEK Zip $5.5 \mathrm{~mm}$, Stryker). ${ }^{6}$ It should also be noted that the surface area to volume ratio of all-suture anchors is greater than traditional anchors because of their smaller size. ${ }^{12}$ Previous work has shown that the smaller all-suture anchors have greater fixation 'per unit area' of soft tissue, and therefore have a relatively greater fixation per unit of volume. ${ }^{12}$

Despite this, recent work from Pfeiffer et al ${ }^{9}$ suggests that, within a canine model, an all-suture anchors promote cavity formation secondary to foreign-body reaction, with resulting expansion of the initial drill tunnel. On the other hand, traditional anchors were found to maintain the initial drill tunnel size. The relative bioincompatibility of all-suture anchors could predicate weaker load-to-failure values after long-term implantation. However, this concept requires further study.

Generally speaking, the findings reported in this study are consistent with those reported in the literature. Ultimately, all anchors aim for minimum gap formation and maximum tensile strength. The maximum pull-out strength values reported are comparable with those of previous studies which have performed similar tests using all-suture anchors, with slight variations in materials and methodology $.6,18,19$

In a bovine humeral model, Galland et al ${ }^{6}$ found that although traditional anchors had higher mean tensile strength and lower elongation values at failure, there was no statistical difference between those and all-suture anchors. These findings are echoed by Mazzocca et al ${ }^{18}$ who found, in a glenoid labral repair model in human bone, that no difference could be demonstrated in ultimate load to failure and displacement at ultimate failure (anchor pull-out) between the all-suture and traditional anchors, despite greater mean values in the former group (171.9 N versus $146.0 \mathrm{~N}$, respectively). They also found that traditional anchors required significantly higher loads to achieve $2 \mathrm{~mm}$ of labral displacement than did all-suture anchors (84.1 $\mathrm{N}$ versus $39.2 \mathrm{~N}$ ).

Of note, the anchors in the current study had a variety of failure mechanisms, typifying the difference in 
construction between all-suture and traditional anchors (Table I). All-suture anchors failed predominantly by anchor pull-out (95\%) which can be attributed to construct failure. This is in contrast to the traditional anchors, which failed by a combination of suture failure, anchor pull-out or eyelet breaking, consistent with what has been previously reported.by others. ${ }^{20}$

With regard to gap formation of the construct, we found that the plastic control anchor had a mean total displacement of $19.7 \mathrm{~mm}$ which is in keeping with work by Rossouw et al, ${ }^{19}$ who demonstrated a mean displacement of $18 \mathrm{~mm}$ in a similar model, but with a metal anchor RC repair. Furthermore, in a complete human cadaveric RC repair model, a standard single-row metal anchor repair demonstrated up to $7.64 \mathrm{~mm}$ (standard deviation 3.74) gap formation after 200 cycles, suggesting the value attained for a simple one-anchor placement is accurate. ${ }^{21}$

Although translation to clinical performance should be managed cautiously, displacement is considered a surrogate for in vivo gap formation post-implant and this could indicate greater instability in the post-operative patient rehabilitation process compared with traditional anchors. Our study showed greater than previously reported gap formation, and this is likely to be due to the specific experimental set up, though it should be noted that the consistency of the construct design and experimental procedures facilitated comparisons of gap formation in implants tested.

Reported displacement in our paper, as in previous studies, is not constrained to movement of the anchor underneath the cortex, but of the whole construct, including the suture material, slippage in the suture grip, and the movement of the bone within the platform (although this was minimal). ${ }^{9}$ Of these, the greatest potential source for artefact came from the suture material itself. Hurwit et a ${ }^{22}$ showed variation within UHMWPE suture material mechanical properties. The group found that by studying initial extension and creep (static and dynamic) as surrogates for initial and time-dependent gap formation, in addition to other mechanical parameters such as stiffness of the sutures, more appropriate determinants of suture performance could be measured. ${ }^{22} \mathrm{~A}$ mean of up to $4.74 \mathrm{~mm}$ of initial extension was observed in one suture and up to $1.52 \mathrm{~mm}$ of extension was observed during static creep in varying UHMWPE sutures. In another study, an in vivo canine model developed by Pfeiffer et $\mathrm{al}^{9}$ compared all-suture with traditional anchors and their findings corroborated with those of this study, reporting that all-suture anchors undergo greater displacement during cyclical loading at ultimate load (mean $13.7 \mathrm{~mm}$ versus $3.2 \mathrm{~mm}$ ).

We argue that displacement as measured within the whole system is a relevant measure because it is representative of gap formation, which is observed in the tissue-anchor construct in vivo, despite the fact that the displacement value does not solely reflect subcortical displacement.

There are limitations to this study. It was performed on cadaveric human humeral heads and was not representative of in vivo conditions with regard to temperature and the proximal suture-tendon interface. Intra- and interdonor variation was not determined by bone density, although reasonable attempts, such as utilising a variety of laterality of humeral heads of different aged cadavers in combination with separate anchor brands to ensure matched sample distributions, to control for this were employed.

Variation in number of UHMWPE sutures could affect the tensile strength of suture anchors, and not all anchors contained the same number of sutures. This limitation of the methodology is somewhat imposed by variations in product design. Furthermore, variations in suture braiding could also affect the tensile strength of anchors, however, with the majority of all-suture anchors failing by anchor pull-out rather than suture failure (95\%), this limitation has minimal influence on the results obtained.

The methodology employed for this study aimed to take the most relevant aspects of previous studies, highlighted by the cyclical loading regime. Unlike previous studies which applied load in line with angle of insertion of the anchor, this study aimed to replicate the direction of force of the RC, which is applied in vivo. However, a unidirectional tensile force through multiple sutures remains incompletely representative of multi-suture cuff repairs in which sutures exert multidirectional tension on the anchor. As a result, findings from this study cannot be directly compared with other work, especially where direct axial loading was performed.

Although this test is not wholly representative of implant performance, the findings can guide clinicians as to implant choice with regard to the mechanical properties of all-suture anchors. The number of anchors tested was consistent with previous work and adequate to determine statistically significant variance; however, a strict left/right pair-wise analysis could not be conducted. $7,9,18,20$

Future work should involve representative repair models comparing all types of all-suture anchors, and add evidence to the suggestion that there are differences between all-suture and traditional anchors. It could be shown that all currently marketed anchors produce an acceptable amount of strength in repair to withstand forces required in the post-operative period (there is currently no published literature on the minimum strength of fixation required for appropriate repair), ${ }^{6}$ although failure is typically by cheese-wiring or gap formation in the longer term.

In the context of traditional anchors, early post-operative anchor movement is a relatively rare complication, reported 
as $0.1 \%$ in one study. ${ }^{23}$ As there has been greater suggested displacement in all-suture anchors in this and other studies, there is cause to consider large-scale follow-up studies of patients with all-suture anchors, especially in the immediate post-operative period.

In conclusion, this study showed that failure load, total displacement, and failure mechanism vary significantly between some all-suture anchors used for RC tear repair. All-suture anchors were found to have lower failure loads compared with a commonly used, traditional anchor. As anchors were not tested in a representative repair model, it is not possible to directly translate findings to a clinical setting. However, intrinsic comparisons in this work give further information to the surgeon as they consider which is the most appropriate implant for a rotator cuff repair procedure in addition to factors such as volume of bone stock removed, likely activity levels of the patient and probability of revision.

\section{Supplementary material}

$\dddot{0}$ A description of the cyclical loading protocol used to measure maximum tensile strength and displacement of anchors can be found alongside the online version of this article at www.bjr.boneandjoint.org.uk

\section{References}

1. Bellumore Y, Mansat M, Assoun J. Results of the surgical repair of the rotator cuff. Radio-clinical correlation. Rev Chir Orthop Reparatrice Appar Mot 1994;80:582-594. (In French)

2. Galatz LM, Ball CM, Teefey SA, et al. The outcome and repair integrity of completely arthroscopically repaired large and massive rotator cuff tears. J Bone Joint Surg [Am]2004;86-A:219-224.

3. Barber FA. Complications of Biodegradable Materials: Anchors and Interference Screws. Sports Med Arthrosc 2015;23:149-155.

4. Barber FA, Herbert MA, Hapa 0, et al. Biomechanical analysis of pullout strengths of rotator cuff and glenoid anchors: 2011 update. Arthroscopy 2011;27:895-905.

5. Barber FA, Herbert MA. Cyclic loading biomechanical analysis of the pullout strengths of rotator cuff and glenoid anchors: 2013 update. Arthroscopy 2013;29:832-844.

6. Galland A, Airaudi S, Gravier R, et al. Pullout strength of all suture anchors in the repair of rotator cuff tears: a biomechanical study. Int Orthop 2013;37:2017-2023.

7. Goschka AM, Hafer JS, Reynolds KA, et al. Biomechanical comparison of traditional anchors to all-suture anchors in a double-row rotator cuff repair cadaver model. Clin Biomech (Bristol, Avon) 2015;30:808-813

8. Hecker AT, Shea M, Hayhurst J0, et al. Pull-out strength of suture anchors for rotator cuff and Bankart lesion repairs. Am J Sports Med 1993;21:874-879.

9. Pfeiffer FM, Smith MJ, Cook JL, Kuroki K. The histologic and biomechanical response of two commercially available small glenoid anchors for use in labral repairs. J Shoulder Elbow Surg 2014;23:1156-1161.
10. No authors listed. ICONIX All Suture Anchor with IntelliBraid Technology. http://textlab.io/doc/9350695/iconix-brochure—stryker-sports-medicine (Date last accessed 20 January 2017).

11. No authors listed. Smith \& Nephew. Q-FIX All-suture implants. https://www.smithnephew.com/global/assets/pdf/products/surgical/sportsmedicine/qfix_brochure_ a1141b.pdf. (date last accessed 20 January 2017).

12. Jost PW, Khair MM, Chen DX, et al. Suture number determines strength of rotator cuff repair. J Bone Joint Surg [Am]2012;94-A:e100.

13. Wilson CL. Pathologic study of degeneration and rupture of the supraspinatus tendon. Arch Surg 1943;47:121.

14. Nightingale EJ, Allen CP, Sonnabend DH, et al. Mechanical properties of the rotator cuff: response to cyclic loading at varying abduction angles. Knee Surg Sports Traumatol Arthrosc 2003;11:389-392.

15. Burkhart SS, Diaz Pagàn JL, Wirth MA, Athanasiou KA. Cyclic loading of anchor-based rotator cuff repairs: confirmation of the tension overload phenomenon and comparison of suture anchor fixation with transosseous fixation. Arthroscopy 1997; 13:720-724.

16. Burkhart SS, Johnson TC, Wirth MA, Athanasiou KA. Cyclic loading of transosseous rotator cuff repairs: tension overload as a possible cause of failure. Arthroscopy 1997:13:172-176.

17. Rupp S, Georg T, Gauss C, et al. Fatigue testing of suture anchors. Am J Sports Med 30:239-247.

18. Mazzocca AD, Chowaniec D, Cote MP, et al. Biomechanical evaluation of classic solid and novel all-soft suture anchors for glenoid labral repair. Arthroscopy 2012;28:642-648

19. Rossouw DJ, McElroy BJ, Amis AA, Emery RJH. A biomechanical evaluation of suture anchors in repair of the rotator cuff. J Bone Joint Surg [Br] 1997;79-B:458-461.

20. Meyer DC, Fucentese SF, Ruffieux K, et al. Mechanical testing of absorbable suture anchors. Arthroscopy 2003;19:188-193.

21. Kim DH, Elattrache NS, Tibone JE, et al. Biomechanical comparison of a singlerow versus double-row suture anchor technique for rotator cuff repair. Am J Sports Med 2006;34:407-414

22. Hurwit D, Fanton G, Tella M, et al. Viscoelastic properties of common suture material used for rotator cuff repair and arthroscopic procedures. Arthroscopy 2014;30:1406-1412

23. Skaliczki G, Paladini P, Merolla G, et al. Early anchor displacement after arthroscopic rotator cuff repair. Int Orthop 2015;39:915-920.

\section{Funding Statement}

The University of Oxford has received an NIHR grant which is related to this article.

Author Contributions

N. N. Nagra: Designed the study, Performed experiments, Subsequent data analysis, Drafted the paper.

N. Zargar: Designed the study, Performed experiments, Subsequent data analysis, Co-drafted the paper.

Co-drafted the paper.
R. D. J. Smith: Performed experiments, Assisted with data analysis, Co-drafted the paper.

A. J. Carr: Designed and supervised the study, Co-drafted the paper.

ICMJE Conflicts of Interest

None declared

(c) 2017 Nagra et al. This is an open-access article distributed under the terms of the Creative Commons Attributions licence (CC-BY-NC), which permits unrestricted use, distribution, and reproduction in any medium, but not for commercial gain, provided the original author and source are credited. 